K. Eveslage

Pflegediagnosen: praktisch und effizient 
K. Eveslage

\section{Pflegediagnosen: praktisch und effizient}

Mit 20 Abbildungen und 39 Tabellen

Springer 


\section{Karin Eveslage}

Landsberger Allee 137

10369 Berlin

ISBN-10 3-540-25578-8 Springer Medizin Verlag Heidelberg

ISBN-13 978-3-540-25578-9 Springer Medizin Verlag Heidelberg

Bibliografische Information der Deutschen Bibliothek

Die Deutsche Bibliothek verzeichnet diese Publikation in der Deutschen Nationalbibliografie;

detaillierte bibliografische Daten sind im Internet über <http://dnb.ddb.de> abrufbar.

Dieses Werk ist urheberrechtlich geschützt. Die dadurch begründeten Rechte, insbesondere die der Übersetzung, des Nachdrucks, des Vortrags, der Entnahme von Abbildungen und Tabellen, der Funksendung, der Mikroverfilmung oder der Vervielfältigung auf anderen Wegen und der Speicherung in Datenverarbeitungsanlagen, bleiben, auch bei nurauszugsweiser Verwertung, vorbehalten. Eine Vervielfältigung dieses Werkes oder von Teilen dieses Werkes ist auch im Einzelfall nur in den Grenzen der gesetzlichen Bestimmungen des Urheberrechtsgesetzes der Bundesrepublik Deutschland vom 9. September 1965 in der jeweils geltenden Fassung zulässig. Sie ist grundsätzlich vergütungspflichtig. Zuwiderhandlungen unterliegen den Strafbestimmungen des Urheberrechtsgesetzes.

\section{Springer Medizin Verlag}

springer.de

(c) Springer Medizin Verlag Heidelberg 2006

Printed in Germany

Die Wiedergabe von Gebrauchsnamen, Handelsnamen, Warenbezeichnungen usw. in diesem Werk berechtigt auch ohne besondere Kennzeichnung nicht zu der Annahme, dass solche Namen im Sinne der Warenzeichenund Markenschutz-Gesetzgebung als frei zu betrachten wären und daher von jedermann benutzt werden dürften.

Produkthaftung: Für Angaben über Dosierungsanweisungen und Applikationsformen kann vom Verlag keine Gewähr übernommen werden. Derartige Angaben müssen vom jeweiligen Anwender im Einzelfall anhand anderer Literaturstellen auf ihre Richtigkeit geprüft werden.

Planung: Barbara Lengricht, Berlin

Projektmanagement: Dr. Ulrike Niesel, Heidelberg

Lektorat: Ute Villwock, Heidelberg

Titelbild und Design: deblik, Berlin

SPIN 11008040

Satz: Stürtz GmbH, Würzburg

Druck und Bindung: Stürtz GmbH, Würzburg 


\section{Geleitwort - Walter Grünzweig}

Einer der häufigsten Vorwürfe gegen die sogenannte »Globalisierung« ist, dass sie die spezifischen kulturellen Traditionen eines Landes nicht berücksichtigt - ja sogar gefährdet. Begriffe wie »McDonaldisierung « oder »Coca-Colonisation« deuten nicht bloß auf die Vernichtung kultureller Vielfalt, sondern verweisen auch gleich auf den angeblichen Bösewicht in diesem Drama des Niedergangs, nämlich die Vereinigten Staaten. Diese Verschwörungstheorien unterstellen den USA, ihre Interessen auch durch Systeme und Sprachregelungen aller Art zu wahren und zu sichern.

Die internationale Propagierung der »NANDA-Klassifikationen « wird für viele Beobachter ein weiterer Beweis für den Willen der letzten verbliebenen Supermacht sein, auch den pflegewissenschaftlichen Bereich in ihrem Sinn zu kolonisieren und zu »amerikanisieren«. Die Homepage der vormaligen »North American Nursing Diagnosis Association«, jetzt »NANDA International «, betont denn auch den internationalen Anspruch auf Gültigkeit ihres Klassifikationssystems, das insbesondere durch die elektronische Datenverarbeitung und seine fast universelle Verwendung in der internationalen Pflegewissenschaft offenbar geworden ist.

Was jedoch als »Amerikanisierung « erscheint, ist bei genauerem Hinsehen oft nichts anderes als die logische Konsequenz einer Entwicklung, auf die wir uns durch unsere Teilnahme an den technologischen und kulturellen Revolutionen des vergangenen Jahrhunderts - manchmal auf Gedeih und Verderb - eingelassen haben. Die amerikanische Mentalität, die nach dem amerikanischen Publizisten John O'Sullivan von der »Zukünftigkeit« geradezu geprägt ist, hat es in einer solchen auf Wechsel angelegten Situation etwas leichter. Keinesfalls jedoch steht es konservativeren Mentalitäten wie etwa der deutschen, die diesen Optimismus nicht in so hohem Maße teilen können, frei, sich aus solchen Entwicklungen einfach auszuklinken.

Vielleicht hilft es, sich darüber klar zu werden, dass das U.S.-amerikanische Denken einen grundsätzlich emanzipatorischen - und damit subversiven - Zug aufweist. Es stellt klassische Hierarchien in Frage. Nicht umsonst hat Karin Eveslage in ihrer Darstellung so häufig literarische Texte zitiert. Denn in solchen Texten werden Phänomene, Probleme und Zusammenhänge präsentiert und benannt, für die wir zunächst keine Namen und keine Begriffe haben. Es sind die Schriftsteller, die dem Menschen sprachlichen Zugang zu Neuem ermöglichen. Erst wer etwas Neues sagen kann, vermag es auch zu denken und vielleicht auch zu tun.

In diesem Sinne ist das Projekt dieses Buchs schöpferisch und emanzipatorisch. Die Pflegewissenschaft, und hier besonders die amerikanische, der vergangenen fünfzig Jahre hat die Menschen gelehrt, Krankheit nicht bloß medizinisch (durch den heilenden Akt) zu konzeptualisieren, sondern die pflegerischen Prozesse als gleichberechtigt und komplementär zu verstehen. Sie hat uns ein alternatives Vokabular gegeben, mithilfe dessen wir die physischen und mentalen Zustände des Menschen alternativ beschreiben und verstehen können. Die internationalen NANDA-Standards geben den Pflegenden überall auf der Welt die Möglichkeit, sich über ihre Arbeit zu verständigen und deren Bedeutung auch öffentlich darzustellen.

Natürlich erschöpft sich das humanistische Anliegen der Pflege nicht in den (gegenwärtig) 172 von der NANDA definierten Pflegediagnosen und es ist ebenso klar, dass sie 
als Vehikel für Kommerzialisierung, Konkurrenzdenken und Ökonomisierung im Gesundheitswesen (miss)braucht werden können und werden. Aber notwendige gesellschaftliche Änderungen werden nicht durch Ausklammern und Ablehnung erreicht, sondern durch aktive Teilnahme aller an solchen Reformen interessierter Kräfte. Eine international anerkannte Systematik kann der Pflegewissenschaft helfen, ihren Forderungen Gehör zu verleihen und damit zu einer gerechteren Gesellschaft im globalen Maßstab beizutragen.

Walter Grünzweig

Professor für amerikanische Literatur und Kultur, Universität Dortmund Adjunct Professor, School of Nursing, University of Pennsylvania 


\section{Vorwort}

"Eine Pflegediagnose ist eine klinische Beurteilung der Reaktion eines Individuums, einer Familie oder einer Gemeinde/Gemeinschaft auf aktuelle und potenzielle Gesundheitsprobleme/Lebensprozesse. Pflegediagnosen bilden die Grundlage zur Auswahl von Pflegeinterventionen, um Ergebnisse zu erreichen, für die Pflegende verantwortlich sind.«

Diese Definition der NANDA (North American Nursing Diagnoses Association) beschreibt den breiten Kontext, in dem Pflegende den Betreuungsprozess des Patienten gestalten können.

Pflegediagnosen sind als etwas sich in der Entwicklung Befindendes zu begreifen. Sie stammen ursprünglich aus Nordamerika. Dennoch oder gerade deshalb ist es unumgänglich, sich mit ihrer internationalen Entwicklung auseinanderzusetzen. Die zunehmende Ökonomisierung zwingt auch Pflegende, ihre Leistungen in Ergänzung zu den medizinischen Interventionen begründet darzustellen und ihren Qualitätsanspruch zu formulieren.

Die Diskussion, ob Pflegediagnosen ein geeignetes Mittel sind, lässt sich nicht durch bloßes Theoretisieren zum Ergebnis führen. Die tägliche Praxis muss zeigen, dass Pflegediagnosen die individuelle Pflege für alle Beteiligten (Leistungsempfänger, Leistungserbringer und Kostenträger) effektiver, effizienter und transparenter werden lässt.

Dabei soll die bedürfnisorientierte Betreuung des Patienten als ein gemeinsames Anliegen im interdisziplinären Team verstanden und auch gelebt werden. Knapper werdende Ressourcen zwingen die verschiedenen Berufsgruppen, die Behandlungsprozesse als ein gemeinsames Anliegen zu begreifen, sie gemeinsam zu überdenken und zu optimieren. Eine hohe Betreuungsqualität, die auch von den Patienten als solche empfunden wird, stärkt das Ansehen der jeweiligen Einrichtung und kann sich in Folge auch auf die Erlössicherung der Institution auswirken.

Die NANDA-Pflegediagnosen sind als internationale Klassifikation am weitesten verbreitet und werden am häufigsten praktiziert. Die Erfahrung hat gezeigt, dass sie auch im deutschsprachigen Raum anwendbar sind. Bei längerer Erfahrung im Umgang mit den Pflegediagnosen wird deutlich, dass sich in der NANDA-Klassifikation durchaus ein Anspruch auf Berücksichtigung in der G-DRG-Systematik (German-Diagnoses Related Groups) verbirgt, auch wenn dieser zurzeit noch nicht direkt geltend gemacht werden kann.

Die G-DRG-Systematik wird als ein lernendes System präsentiert. Damit erhalten Pflegende die Chance, ihre Ansprüche im Interesse einer hohen Betreuungsqualität transparent zu machen. Voraussetzung dafür ist eine breite und forcierte Einführung der NANDA-Pflegediagnosen überall dort, wo Patienten Anspruch auf indikationsgerechte Pflegeleistungen haben. Es geht nicht primär um die Frage, was der Gesetzgeber für die Pflege tun kann, sondern vielmehr darum, was die Pflegenden dem Gesetzgeber an die Hand geben, damit ihr Anliegen mit einem Gesetz unterstützen werden kann. Einer Berücksichtigung von Pflegediagnosen zur Kosten- und Erlöstransparenz muss zunächst eine breit angelegte Initiative zur Einführung dieser Diagnosen vorangehen.

Pflegewissenschaftliche Entwicklungen können so - eingebunden in den diagnostischen Prozess und in betriebswirtschaftliche Erfordernisse - in den Klinikalltag integriert werden. Dabei haben zwischenmenschliche Kontakte und organisatorische Abläufe einen 
hohen Stellenwert, denn der Patient beurteilt die Qualität der Behandlung nach seinen Maßstäben und nach seinen Empfindungen. In diesem Prozess erfüllen Pflegepersonen eine bedeutende Rolle. Sie verbringen die meiste Zeit mit den Patienten und ihren Angehörigen. Sie kennen ihre Empfindungen, ihre individuellen Probleme und nehmen zuerst Veränderungen wahr. Auch die organisatorischen Abläufe werden im Stationsalltag hauptsächlich vom Pflegepersonal gesteuert. Durch die Veränderungen im Gesundheitswesen und die stärker werdenden ökonomischen Zwänge erhalten Pflegende die Chance, ihre Arbeitsfelder neu und selbstbewusst zu definieren. Sie können den diagnostischen Prozess als ihre originäre Aufgabe verstehen und gestalten lernen.

Ein solcher Paradigmenwechsel gelingt, wenn eine moderne Führungsphilosophie diese Entwicklung flankiert und wenn das Führungspersonal bereit ist, motivierte Mitarbeiter im diagnostischen Prozess so zu unterstützen, dass die Pflegediagnosen in der Gestaltung des Behandlungsprozesses zu einer unumgänglichen Größe werden.

\section{Danksagung}

Mein Dank gilt den Mitarbeitern der Charité Universitätsmedizin Berlin, durch deren Neugier und kritisches Engagement dieses Buch erst möglich wurde. Insbesondere bedanke ich mich bei Ramona Schumacher, die während ihrer Zeit als Pflegedirektorin an der Charité mit einem innovativen Führungsstil diesem Projekt viel Gestaltungsraum ließ.

Ich bedanke mich bei den Mitgliedern des Projektteams Susanne Körner, Barbara Thrane und Daniela Hertel, sowie bei allen Multiplikatoren, durch deren Ausdauer und Entschlossenheit die Erprobung der NANDA-Pflegediagnosen gelingen konnte.

Darüber hinaus möchte ich Justus Rademacher und Katrin Gärtner meinen Dank aussprechen, die eine Dokumentationsform durchsetzen konnten, die eine effiziente Abbildung des Pflegeprozesses in der Patientenakte ermöglicht.

Des Weiteren gilt mein Dank allen Stationsleitungen, Abteilungsleitungen, Ärzten und den Mitarbeitern des Medizin Controllings, die diese Arbeit im Erprobungsprozess ebenfalls begleitet und unterstützt haben.

Für die wertvollen Hinweise und die hervorragende Betreuung danke ich ganz herzlich Barbara Lengricht im Springer Verlag, die das Buch auf den Weg gebracht hat, und Ute Villwock für ihre unermüdliche Lektoratstätigkeit.

Berlin

Karin Eveslage 


\section{Einführung}

Dieses Buch richtet sich an diejenigen Mitarbeiter, die am Betreuungsprozess von Patienten beteiligt sind, sei es als Pflegende oder als Führungspersonen. Es soll darüber hinaus all denjenigen eine Hilfe sein, die in den verschiedenen Ausbildungen zukünftige Mitarbeiter in Gesundheitseinrichtungen auf die veränderten Anforderungen vorbereiten, und natürlich auch den Ärzten, denen Pflege mehr bedeutet als nur die Assistenz und Ausführung von ärztlichen Verordnungen. Während der Erprobung ging es primär darum, ein Verständnis für pflegetheoretische Zusammenhänge im Kontext von Betreuungsqualität, Ökonomisierung und Mitarbeiterführung zu entwickeln.

In den nachfolgenden Kapiteln werden Wege aufgezeigt, wie mit Hilfe von Pflegediagnosen hohe Qualitätsansprüche und zunehmende Ökonomisierung in Einklang gebracht werden können. Im Universitätsklinikum Charité wurde im Jahre 2000 mit der Erprobung der NANDA-Pflegediagnosen begonnen. Die Erfahrungen aus einer vierjährigen Projektphase sind in die einzelnen Kapitel eingeflossen.

Arbeitsgrundlage für die Einführung der NANDA-Pflegediagnosen war das Buch von Stefan H, Allmer F, Eberl J (1999, 2000 und 2003) Praxis der Pflegediagnosen. Springer Wien.

Auf eine ausführliche inhaltliche Darstellung der NANDA-Pflegediagnosen wurde verzichtet, da diese bereits in diesem sehr empfehlenswerten Buch beschrieben wurden und in Fachkreisen bekannt sind. Die in den Fallbeispielen verwandten Formulierungen (Pflegediagnosentitel, Ätiologie und Symptome) stammen aus NANDA international (2005) NANDA-Pflegediagnosen. Definition und Klassifikation 2005-2006. Hans Huber, Bern, in der Deutschsprachigen Ausgabe herausgegeben von Jürgen Georg. Die Ziele und Maßnahmen wurden teilweise modifiziert. 


\section{Inhaltsverzeichnis}

\section{Schritt l:}

Voraussetzungen zur praktischen Umsetzung

$1 \quad$ Pflegediagnosen - ihre Bedeutung für das Qualitätsund Risikomanagement sowie die G-DRG-Systematik............. 3

1.1 Definition und historische Entwicklung der NANDA-Pflegediagnosen ......... 4

1.2 Pflegediagnosen und ihre Relevanz für Veränderungsprozesse............. 10

1.2.1 Der Interaktionsprozess als systemischer Ansatz bei der Implementierung

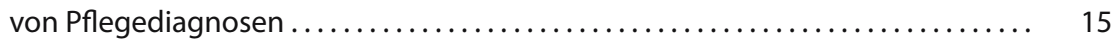

1.2.2 Pflegetheorien und pflegediagnostischer Prozess . ..................... 18

$1.3 \quad$ Individuelle Betreuung und Standardisierung ....................... 29

Literatur ............................................... 32

2 Pflegediagnosen und ihre Bedeutung für die Transparenz des Behandlungsaufwandes (Ralf Hammerich) ............... 33

2.1 Pflegediagnosen als Beitrag zur Erlössicherung im Rahmen der G-DRG-Systematik ..................................... 34

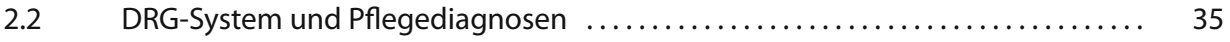

2.2.1 Die historisch bedeutsamen Entwicklungen hin zum G-DRG-System ......... 36

2.2.2 Die Zielsetzung des G-DRG-Systems........................... 37

2.2.3 Die Datenlieferung und Aufarbeitung im InEK ...................... 40

2.2.4 Die Bedeutung von Diagnosen und Prozeduren im DRG-System ............ 41

2.2.5 Der Einfluss von Pflegdiagnosen im aktuellen System 2006 ............... 46

2.3 Pflegediagnosen im Kontext der G-AEP-Kriterien und MDK-Prüfungen ........ 49

2.3.1 Das Sozialgesetzbuch V als Grundlage für die Versorgung kranker Menschen im bundesdeutschen Gesundheitssystem ....................... 50

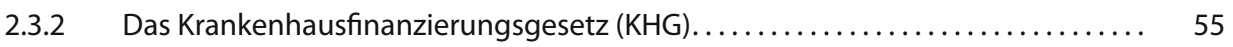

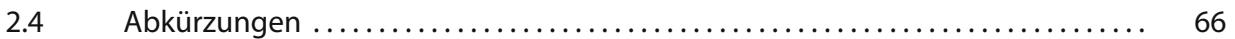

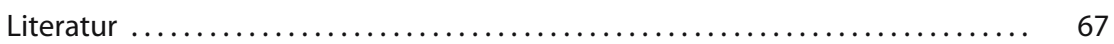

$3 \quad$ Vorbereitung für die Einführung von Pflegediagnosen.............. 69

$3.1 \quad$ Bestimmung der Ist-Situation: Standortbestimmung .................. 70

3.1.1 Evaluation des bisherigen Dokumentationsprozesses.................. 72

3.1.2 Sicherstellung von Ressourcen................................... 74

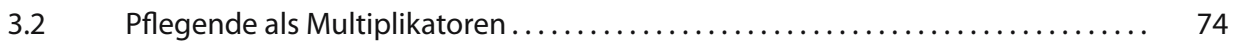

3.2.1 Auswahl von Multiplikatoren ................................. 75

3.2.2 Aufgaben der Multiplikatoren................................. 75

3.3 Vorbereitungen der Multiplikatoren - Qualifikationskonzept............. 78

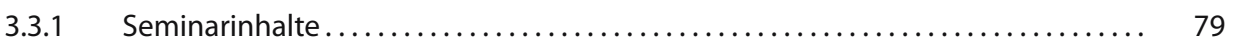

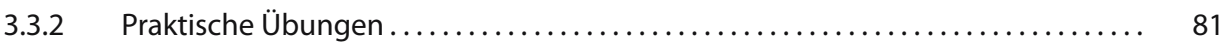

3.3.3 Kontinuierlicher Verbesserungsprozess.............................. 81

Literatur................................................ 82 


\section{Schritt II:}

Pflegediagnosen in der praktischen Umsetzung

$4 \quad$ Aufgaben und Struktur von Pflegediagnosen .................. 85

$4.1 \quad$ Aufgaben von Pflegediagnosen.................................... 86

4.2 Die Struktur der Pflegediagnosen ............................... 87

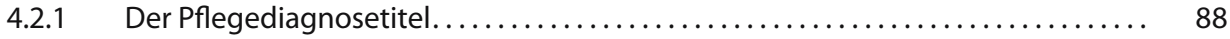

4.2.2 Einflussfaktor/Ätiologie..................................... 88

4.2 .3 Symptom/Kennzeichen..................................... 89

4.2.4 Einteilungsstufen des Selbstständigkeitsgrades ...................... 89

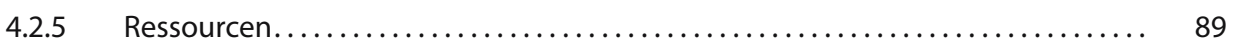

4.3 Richtlinien zur Formulierung von Pflegediagnosen .................... 90

4.3.1 Aktuelle Pflegediagnosen....................................... 90

4.3.2 Hoch-Risikodiagnosen..................................... 92

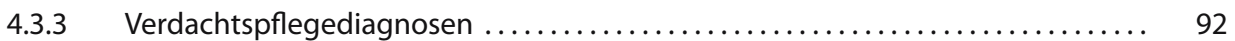

4.3.4 Syndrom-Pflegediagnosen................................... 93

4.3.5 Gesundheitspflegediagnosen/Wellness-Pflegediagnosen................ 93

4.3.6 Potenzielle Komplikationen.................................. 94

4.4 Aufbau eines klinikinternen Kataloges ............................. 99

4.4.1 Die Verbindung von Pflegetheorie und Katalogsystematik ................ 100

Literatur.............................................. 102

$5 \quad$ Pflegediagnosen und diagnostischer Prozess .................... 103

5.1 Kritisches und kreatives Denken im diagnostischen Prozess................. 105

5.2 Der diagnostische Prozess als gemeinschaftliche Aktivität von Patient, Angehörigen und Pflegepersonal ........................... 106

5.2.1 Die Pflegeanamnese als wichtiges Element der Informationssammlung ....... 106

5.2.2 Analysieren, interpretieren und verdichten von Informationen im diagnostischen Prozess ................................... 108

5.2.3 Evaluationsmöglichkeiten im Rahmen von Pflegevisiten................... 109

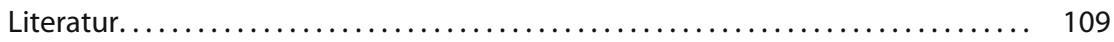

\section{Schritt III:}

\section{Leistungsoptimierung durch Veränderungen} in der Organisation des Behandlungsprozesses und in der Mitarbeiterführung

6 Pflegediagnosen und ihre Bedeutung für neue Organisationsformen im Behandlungsprozess............................. 113

6.1 Case Management als neue Organisationsform ....................... 114

6.1.1 Definition und Zielsetzung. ....................................... 114

6.1.2 Case Management und interprofessionelle Kooperation ................. 116

6.1.3 Potenzielle Instrumente eines Case Managements. . . . . . . . . . . . . . . . . 117

6.1.4 Potenzielle Aufgaben eines Case Managers......................... 120 
6.1.5 Case Management und Pflegediagnosen - Leistungsoptimierung

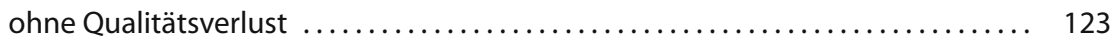

Literatur................................................... 124

$7 \quad$ Kompetenzentwicklung im Rahmen von Personalmanagement........ 125

$7.1 \quad$ Pflegediagnostischer Prozess und Kompetenzentwicklung ............... 126

7.1.1 Kompetenzstufen und Wissensentwicklung im diagnostischen Prozess . ...... 127

7.1.2 Unterschiedliche Kompetenzstufen in der Pflegediagnostik............... 130

7.2 Führungs- und Mitabeiterverhalten im Umgang mit verschiedenen Kompetenzstufen .......................................... 132

7.2.1 Kompetenzentwicklung durch Deregulierung ...................... 133

7.2.2 Kompetenzentwicklung durch Vertrauen.......................... 134

Literatur................................................... 135

\section{Schritt IV:}

Fallbeispiele

$8 \quad$ Potenzielle Varianten von individueller und standardisierter Planung

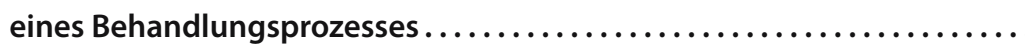

8.1 Musterbeispiel für eine Pflegeplanung nach einer ausgewählten medizinischen Diagnose.

8.2 Musterbeispiel für eine Pflegeplanung nach einer ausgewählten medizinischen Prozedur

8.3 Musterbeispiel für die Integration von Pflegediagnosen in einen klinischen Behandlungspfad ........................... 144

8.3.1 Definition und Zielsetzung. ................................... 144

8.3.2 Dokumentationsinstrumente für einen klinischen Behandlungspfad .......... 151

8.4 Musterbeispiel für die Einarbeitung von Pflegediagnosen

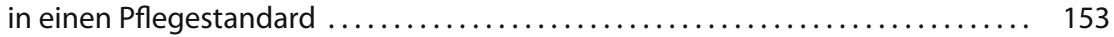

Literatur. .................................................. 153

Stichwortverzeichnis 